\title{
THE MID-INFRARED SPECTRUM OF THE SHORT ORBITAL PERIOD POLAR EF ERIDANI FROM THE SPITZER SPACE TELESCOPE
}

\author{
D. W. Hoard, ${ }^{1}$ Steve B. Howell ${ }^{2}$ Carolyn S. Brinkworth, ${ }^{1}$ David R. Ciardi, ${ }^{3}$ and Stefanie Wachter ${ }^{1}$ \\ Received 2007 July 12; accepted 2007 August 20
}

\begin{abstract}
We present the first mid-infrared (5.5-14.5 $\mu \mathrm{m})$ spectrum of a highly magnetic cataclysmic variable, EF Eridani, obtained with the Infrared Spectrograph (IRS) on the Spitzer Space Telescope. The spectrum displays a relatively flat, featureless continuum. A spectral energy distribution model consisting of a $9500 \mathrm{~K}$ white dwarf, an L5 secondary star, cyclotron emission corresponding to a $B \approx 13 \mathrm{MG}$ white dwarf magnetic field, and an optically thin circumbinary dust disk is in reasonable agreement with the extant Two Micron All Sky Survey (2MASS), Infrared Array Camera (IRAC), and IRS observations of EF Eri. Cyclotron emission is ruled out as a dominant contributor to the infrared flux density at wavelengths $\gtrsim 3 \mu \mathrm{m}$. The spectral energy distribution longward of $\sim 5 \mu \mathrm{m}$ is dominated by dust emission. Even longer wavelength observations would test the model's prediction of a continuing gradual decline in the circumbinary diskdominated region of the spectral energy distribution.
\end{abstract}

Subject headings: novae, cataclysmic variables — stars: individual (EF Eri) — stars: low-mass, brown dwarfs

Online material: color figure

\section{INTRODUCTION}

Cataclysmic variables (CVs) are interacting binary stars containing a white dwarf (WD) primary and a low-mass secondary (Warner 1995). The evolution of these binaries is believed to proceed as follows (see Howell et al. 2001): after a common-envelope phase following the post-main-sequence evolution of the WD progenitor, the low-mass secondary star eventually (over)fills its Roche lobe, and the binary commences mass transfer. Due to angular momentum losses, primarily via magnetic braking and gravitational radiation, the two component stars move closer together over time; that is, their orbital period decreases. For the oldest CVs, the orbital periods are very short (near 80 minutes), and the secondary stars are very low mass, being $\sim 0.06 M_{\odot}$ stars or lower mass degenerate brown dwarf-like objects.

EF Eridani contains a strongly magnetic $\mathrm{WD}(B \approx 13-14 \mathrm{MG}$; Wheatley \& Ramsay 1998; Howell et al. 2006a; Beuermann et al. 2007), making it a member of the polar class of CVs (named for the highly polarized nature of their emitted light). Unlike CVs containing nonmagnetic WDs, polars have no accretion disks (instead, accretion proceeds directly from the inner Lagrangian point onto the magnetic field lines of the WD), and generally do not undergo dwarf-nova-type (i.e., disk instability) outbursts. They do, however, experience periods of normal mass transfer from the secondary star (high states) interspersed with times when this accretion flow stops or is significantly decreased (low states), possibly related to stellar activity on the secondary star. EF Eri has been in a low state for the past 10 years (Howell et al. 2006a). Interestingly, recent high-energy observations in the UV (Szkody et al. 2006) and X-rays (Schwope et al. 2007) have shown that EF Eri still has an $\sim 20,000 \mathrm{~K}$ hot spot remaining on the surface of its $\sim 10,000 \mathrm{~K} \mathrm{WD}$, even after a decade of very low mass accretion $\left(\dot{M} \sim 10^{-14} M_{\odot} \mathrm{yr}^{-1}\right)$. This hot region is presumably at or near the active accretion pole, which is best modeled as a nonuniform spot covering $10 \%-20 \%$ of one side of the WD (Beuermann et al. 2007).

\footnotetext{
1 Spitzer Science Center, California Institute of Technology, Pasadena, CA 91125.

2 WIYN Observatory and National Optical Astronomy Observatory, Tucson, AZ 85719 .

Michelson Science Center, California Institute of Technology, Pasadena, CA 91125 .
}

Our initial Spitzer Space Telescope (Werner et al. 2004) photometric observations of a small sample of polars using the Infrared Array Camera (IRAC; Fazio et al. 2004) included EF Eri as the brightest sample member, and revealed a nearly flat mid-IR (3.6-8 $\mu \mathrm{m}$ ) flux density level near $700 \mu \mathrm{Jy}$ (Howell et al. 2006b; Brinkworth et al. 2007). In Howell et al. (2006b), we showed that the IRAC spectral energy distributions (SEDs) of EF Eri and three other polars with similar orbital periods had flux densities in excess of that produced by the two component stars alone. It was concluded that the best candidate for the excess emission was a circumbinary dust disk with an inner-edge temperature $\left(T_{\text {in }}\right)$ near $800 \mathrm{~K}$. In Brinkworth et al. (2007, hereafter B07), we used these same data, as well as IRAC observations of two additional polars (making a total of seven systems), to examine a larger suite of more sophisticated SED models. For EF Eri, we found that the most plausible way to produce the observed bright $8 \mu \mathrm{m}$ flux density, without exceeding the observed flux densities at shorter wavelengths, was via a geometrically thin, optically thick circumbinary disk (CBD) with $T_{\text {in }} \approx 650 \mathrm{~K}$; however, we could not completely rule out the possibility that at least some of the long-wavelength flux density in the SED is due to cyclotron emission. The B07 model results were generally consistent with the need for a prominent cyclotron emission component to explain the near-IR (e.g., Two Micron All Sky Survey [2MASS]) portion of the EF Eri SED.

The Spitzer photometric observations alone could not provide any further clarification as to the exact origin of the $3.6-8 \mu \mathrm{m} \mathrm{SED}$, as they had limited wavelength resolution and coverage. Consequently, those data were not able to strongly constrain models for the origin of the observed mid-IR flux density of EF Eri (as described in more detail in B07). Therefore, in an effort to better understand the true nature and extent of the mid-IR emitting source(s) in EF Eri, we obtained a spectrum spanning 5.5-14.5 $\mu \mathrm{m}$ using the Infrared Spectrograph (IRS; Houck et al. 2004) on Spitzer.

\section{OBSERVATIONS AND DATA PROCESSING}

\subsection{Mid-IR Spectrum}

Our spectroscopic observations of EF Eri were obtained using the "Short-Low" module of the IRS, which covers 5.2-8.7 $\mu \mathrm{m}$ in second order (SL2) and 7.4-14.5 $\mu \mathrm{m}$ in first order (SL1) at a 


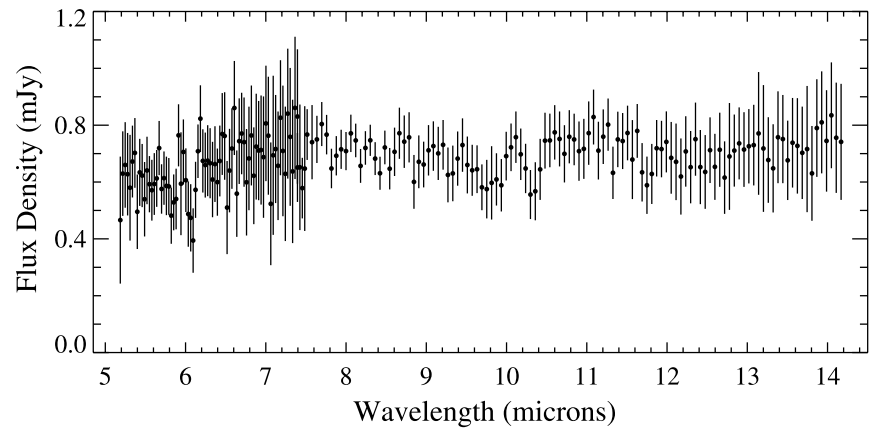

FIG. 1.-Mid-IR spectrum of EF Eri from the Spitzer Space Telescope (with $1 \sigma$ error bars).

resolution of $R \sim 60-120$. A third order (the SL3 "bonus" spectrum) is obtained with each SL2 observation; it spans 7.4-8.6 $\mu \mathrm{m}$ and is primarily used to ensure that the flux calibration from SL2 to SL1 is consistent. (In our case, all three orders were in excellent agreement in the overlapping wavelength region, so we did not apply any offsets between orders, and used the data from all three orders in constructing a final spectrum; see below.) We obtained four cycles of $240 \mathrm{~s}$ each for both SL1 and SL2 (i.e., a total of 16 individual exposures after counting the two nod positions obtained in each cycle). The corresponding AOR Key is 17052928.

We used the Spitzer Science Center post-BCD software SPICE (ver. 1.4.1 $)^{4}$ to extract the IRS spectra from the two-dimensional images. The input images consisted of the four S15.3.0 pipelinecombined post-BCD images (i.e., the $*$ bksub fits files), each of which is constructed from four appropriately backgroundsubtracted, masked, and co-added subexposures to give two images each for the SL1 and SL2+SL3 orders (i.e., one image per order at each nod position). We extracted the spectra from these two-dimensional images using the optimal extraction algorithm with the standard aperture width. This results in two extracted spectra (one for each nod position) spanning the three SL orders.

Next, we performed a weighted average of the two (or three) points at each wavelength from the two nod spectra to obtain a preliminary average spectrum. We calculated the weighted mean, $\left\langle f_{w}\right\rangle$, and standard deviation of the weighted mean, $\sigma_{w, \text { avg }}$, of the flux density over the full wavelength range for the preliminary average spectrum. Then, we rejected any point in an individual nod spectrum that was more than $3 \sigma_{w}$, avg away from $\left\langle f_{w}\right\rangle$ when the corresponding point(s) at the same wavelength in the other nod was not (i.e., an outlier rejection). Finally, we recalculated the average spectrum using the outlier-rejected nod spectra, via a weighted average when two or more data points were available for a given wavelength, or using the remaining data point when only one survived rejection.

The IRS spectrum of EF Eri is shown in Figure 1. It is characterized by a generally flat continuum with a slight dip at the shortwavelength end. There are no obvious emission features, and only a few potential absorption features. However, we note that the IRS was designed to achieve high sensitivity at the cost of reduced dynamic range; hence, great care must be used in the interpretation of weak spectral features, ${ }^{5}$ especially in spectra of faint targets such as EF Eri. The spectrum of EF Eri used in this work has not been scaled from its original flux calibration; the excellent agreement in flux density between the IRAC photometric points and the IRS spectrum (see $\S 2.2$ and Fig. 2), as well as the smooth

\footnotetext{
${ }^{4}$ See http://ssc.spitzer.caltech.edu/postbcd/spice.html.

5 See the IRS chapter of the Spitzer Observer's Manual, available at http://ssc .spitzer.caltech.edu/documents/som/.
}

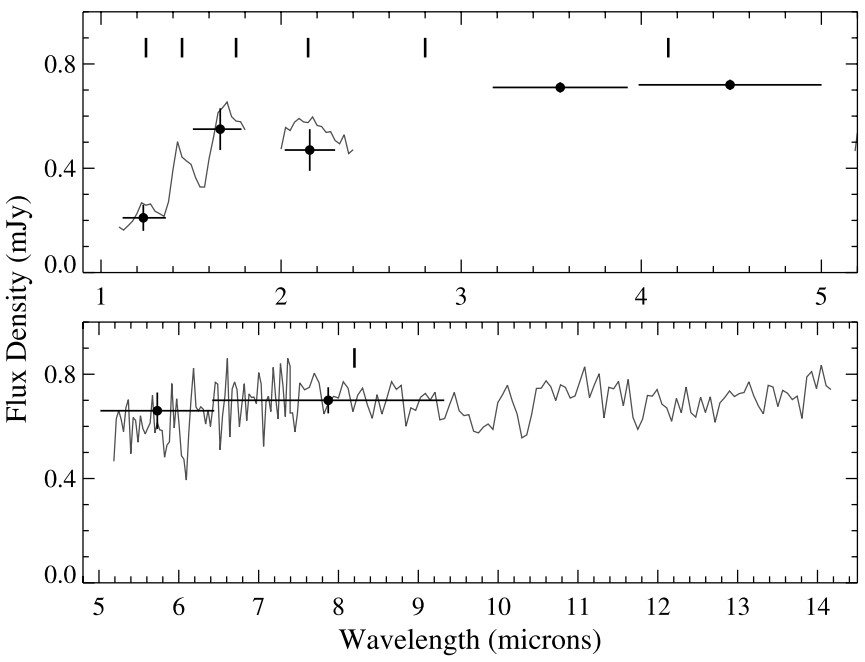

FIG. 2.-IR observations of EF Eri. The photometric data from Brinkworth et al. (2007) are shown as filled circles; from short to long wavelengths, these are 2MASS $J, H$, and $K_{s}$, and IRAC channels $1-4$. Error bars on all of the photometric points are the $1 \sigma$ photometric uncertainties in the $y$-direction (these are smaller than the plotting symbols for IRAC channels 1 and 2), and the widths of the photometric bands in the $x$-direction. The near-IR spectrum (taken from Fig. 1 of Harrison et al. 2007) and IRS mid-IR spectrum of EF Eri are shown as solid lines (error bars have been omitted for clarity). The vertical hashmarks show the cyclotron harmonic peaks for $n=2-7$ (top, from right to left) and the cyclotron fundamental (bottom) for the model shown in Fig. $3 b$ and discussed in $\S 3.2 .4$. Note the different wavelength scales in the two panels.

transition from the SL2 to SL1 data sections (at $\lambda \approx 7.5 \mu \mathrm{m}$ ), attests that the overall continuum shape of the spectrum is reliable. Consequently, our analysis presented here will rely on looking at the gross properties of the spectrum as a whole (i.e., the continuum shape and flux density level), rather than focusing on specific features with low statistical probability of being real.

\subsection{Other Data}

We also utilized the 2MASS and IRAC photometry of EF Eri reported in B07, and the near-IR spectrum shown in Figure 1 of Harrison et al. (2007). Figure 2 shows all of the spectroscopic and photometric data plotted together. Other than the points from IRAC channels 1 and 2 (3.6 and $4.5 \mu \mathrm{m}$, for which there are no overlapping spectroscopic data), all of the photometric data are in excellent agreement with the spectroscopic data. A slight exception to this is the 2MASS $K_{s}$-band point, which is significantly lower in flux density (by more than $1 \sigma$ ) than the near-IR spectrum. This is likely due to the $K$-band variability noted by Harrison et al. (2004), which has been linked to cyclotron emission that varies with orbital phase.

\section{SPECTRAL ENERGY DISTRIBUTION MODELS}

\subsection{The Code}

In B07, we introduced our IR SED modeling code for CVs, which we applied to photometric data for several polars, including EF Eri. We have now made a number of improvements to the modeling code for use here. First, we have modified the code to allow SEDs to be calculated at higher wavelength resolution, which is more appropriate for comparing to spectral data. This modification has little effect on the WD and CBD components, but provides the necessary resolution to resolve cyclotron humps in the cyclotron component. For the models presented here, we have used a wavelength increment of $0.05 \mu \mathrm{m}$ in the range $1-14.5 \mu \mathrm{m}$.

Second, we have modified the handling of the secondary star to accommodate data at longer wavelengths. In B07, the secondary 
star was represented by average 2MASS and IRAC photometry for spectral type templates from Patten et al. (2006). We have now added the capability to combine the 2MASS and IRAC photometry of a single, representative spectral type star from Patten et al. (2006) with the IRS SL spectrum of the same star from Cushing et al. (2006). For the EF Eri models presented here, the secondary star model component (see Table 2) is represented by the L5 star 2MASS J15074769-1627386 (Reid et al. 2000) scaled to a distance of $d=132 \mathrm{pc}($ see $\oint 3.2)$.

Third, we have made a number of minor improvements to the calculation of the optically thin CBD component SED, which is primarily used in this work instead of the optically thick CBD used in B07. In general, the procedure remains as described in B07. The SED of the CBD is obtained by summing the contribution of 1000 annular rings, each of which has the same width and the correct temperature for its average radial distance from the center of mass of the CV [based on the $T \propto(1 / r)^{3 / 4}$ profile explained in more detail in B07]. The volume of a ring increases as its radial distance increases, and we assume equal mass in each ring, which results in rings that are successively less dense at larger radial distances.

We have imposed an upper limit of $1000 \mathrm{~K}$ for the temperature of the inner edge of the CBD. Not only is it difficult to devise a mechanism that would heat the inner edge of the CBD in EF Eri above $1000 \mathrm{~K}$, but dust sublimation (and hence destruction of the CBD) likely occurs at temperatures above 1000-2000 K. Based purely on the WD effective temperature, we would expect the temperature at the radius of the inner edge of the CBD to be $T_{\text {in }} \approx$ $250 \mathrm{~K}$; including the contribution from the secondary star, this temperature increases to $T_{\text {in }} \approx 400-500 \mathrm{~K}$. However, as noted in B07, the upper limit to this temperature is difficult to assess because of the uncertainties in how to properly account for the contributions from the secondary star and accretion luminosity; in addition, the conversion from the ambient temperature of irradiation at a particular radius to temperature of the CBD material at that radius is uncertain and highly dependent on the physical parameters of the CBD and the WD accretion spot(s). Especially considering the evidence from recent UV and X-ray observations (Szkody et al. 2006; Schwope et al. 2007) that there is a significant hot spot on the WD in EF Eri, the temperature at the inner edge of the CBD could be up to several hundreds of $\mathrm{K}$ higher. Effectively, then, we define an "allowed" range of $T_{\text {in }}=400-1000 \mathrm{~K}$.

Finally, and most significantly, we have replaced the purely morphological, template-based cyclotron component from B07 with a physical model based on the formulation discussed and used in, for example, Chanmugam (1980), Thompson \& Cawthorne (1987), Schwope et al. (1990), and references therein. The calculation of the cyclotron SED is influenced by several parameters: the WD magnetic field strength $(B)$, the viewing angle $(\theta)$, the electron temperature $(k T)$, a dimensionless "size" parameter $(\Lambda)$, and a normalization factor $(A)$. None of these parameters is strictly independent of the others; the change in the cyclotron SED produced by adjusting one parameter can usually be offset by adjusting one or more of the other parameters. This makes it difficult to produce a unique solution without having reliable, very narrow constraints for as many of the parameters as possible. We will describe the constraints used to narrow down the possible cyclotron SEDs for EF Eri in $\S 3.2$. In the remainder of this section, we will discuss, in general terms, the manner in which each parameter influences the cyclotron SED.

To first order, the WD magnetic field strength $(B)$ determines the wavelengths at which cyclotron humps appear in the SED for successively higher harmonic numbers. Smaller values of $B$ produce more redshifted humps. However, the wavelengths of the harmonics are also influenced by the electron temperature $(k T)$ and the viewing angle $(\theta)$. Increasing $k T$ or decreasing $\theta$ redshifts the humps. The humps become broader with increasing $k T$, increasing size parameter $\Lambda$ (however, see below for additional effects of changing $\Lambda$ ), or decreasing $\theta$. At very high values of these parameters (especially $k T$ ), the humps are so broad as to be completely overlapping and blended together, effectively forming a humpless pseudocontinuum. At very low values, the cyclotron humps are very narrow, resulting in a cyclotron spectrum that consists of multiple discrete hump profiles with regions of zero intensity between them. The normalization factor $(A)$ is a simple scaling factor given by the ratio of the effective emitting area of the cyclotron component to the square of the distance to the $\mathrm{CV}$.

The size parameter $\Lambda$ deserves additional explanation. Although its value is set in the cyclotron SED calculation as a single number, this parameter actually incorporates several other parameters. It is defined (see Schwope et al. 1990) as

$$
\Lambda=2.01 \times 10^{5}\left(\frac{s}{10^{5} \mathrm{~cm}}\right)\left(\frac{N_{e}}{10^{16} \mathrm{~cm}^{-3}}\right)\left(\frac{3 \times 10^{7} \mathrm{G}}{B}\right),
$$

where $s$ is the geometric path length through the cyclotron emitting region and $N_{e}$ is the electron number density. For a fixed value of $B$, the value of $\Lambda$ is dependent on only the product of $s$ and $N_{e}$. Functionally, larger values of $\Lambda$ correspond to higher optical depths, which influences the harmonic number at which the cyclotron humps transition from being optically thick (at lower harmonics) to optically thin (at higher harmonics). Optically thick cyclotron humps have a truncated, flat-topped appearance compared to the rounded optically thin humps. The value of $\Lambda$ also has a large effect on the relative amplitudes of the cyclotron humps. The lower harmonic, optically thick humps at longer wavelengths rapidly decline in amplitude as harmonic number decreases compared to the optically thin humps at higher harmonics (shorter wavelengths).

\subsection{The Models}

\subsubsection{Distance to EF Eri}

The distance used to scale the model components in this work is the $1 \sigma$ upper limit of a trigonometric parallax-derived distance from Thorstensen (2003), and is approximately halfway between the two possible nominal parallax distance values of 113 and $162 \mathrm{pc}$ reported in that work. We increased the distance from the $105 \mathrm{pc}$ used in B07 (which was based on nonparallax estimates) because it is now clear that cyclotron emission makes a nonnegligible contribution in the $J$ band (see discussion of models below), whereas at the smaller distance, the observed $J$-band flux density was completely accounted for by the WD and secondary star components. The value of $132 \mathrm{pc}$ used here is the minimum distance for which the summed WD, secondary star, and cyclotron emission do not exceed the observed photometric and spectroscopic data at $J$ band.

\subsubsection{Cyclotron Component Constraints}

As noted in $\S 3.1$, our revised cyclotron model component is calculated using a number of non-independent parameters, so it is helpful to constrain, as much as possible, the range of valid parameter space. To that end, we describe in this section the various constraints that we used to limit the cyclotron model parameters.

1. $B$ : The WD magnetic field in EF Eri is frequently derived as $B=13-14$ MG (e.g., Wheatley \& Ramsay 1998; Howell et al. 
2006a; Beuermann et al. 2007). A value of $B$ in this range is consistent with the location of the cyclotron humps in the nearIR spectrum of EF Eri (Harrison et al. 2007; see also Fig. 2). We do not consider the much more complex scenario in which the cyclotron spectrum of EF Eri results from two or more magnetic accretion regions with significantly different field strengths.

2. $k T$ : The near-IR spectrum of EF Eri also provides useful constraints for this parameter. Even after subtracting the WD and secondary star components, there is residual flux between the cyclotron humps. This requires $k T \gtrsim 5 \mathrm{keV}$ (at lower values of $k T$ and for reasonable values of $\Lambda$ [see below], the cyclotron spectrum is composed of discrete humps with zero flux level between them). In addition, for $k T \lesssim 5 \mathrm{keV}$, and for values of $\Lambda$ that reproduce the observed optically thick-to-thin transition and relative hump peak amplitudes, the cyclotron humps are too narrow compared to the observed near-IR spectrum. The fact that discrete cyclotron humps are observed requires $k T \lesssim 20 \mathrm{keV}$.

3. $\theta$ : In the absence of consistent information constraining this parameter, we chose to set $\theta=75^{\circ}$ for all models. In any case, $\theta$ cannot be much lower or higher than this, or the widths and relative amplitudes of the optically thin humps will not match the observed near-IR spectrum.

4. $\Lambda$ : For any given values of the other parameters, $\Lambda$ is the most constrained parameter. This is because only a narrow range of its values will reproduce the observed near-IR spectrum, which shows the $n=4$ harmonic as optically thick, and higher harmonics as optically thin. The value of $\Lambda$ is further fine tuned by matching the relative peak amplitudes of both the optically thick and thin cyclotron humps.

5. $A$ : The main constraint on the scaling parameter is that for a given distance, $A$ should correspond to an effective emitting area of the cyclotron radiation ( $\left.a_{\text {cyc }}\right)$ that is (much) smaller than the projected surface area of the WD.

Most of these constraints are derived from the near-IR spectrum of EF Eri, which shows the cyclotron hump at the $n=4$ harmonic to be flat-topped and much lower in peak amplitude than the humps at the $n \geq 5$ harmonics (see Fig. 2). This indicates that the transition from optically thick to thin cyclotron emission occurs in the vicinity of the $n=4$ harmonic. In particular, satisfying the observed transition from optically thick to thin humps results in a mid-IR contribution of the cyclotron emission that is increasingly negligible at longer wavelengths. That is, the cyclotron component is only important at relatively short IR wavelengths, and does not contribute significantly to the mid-IR region spanned by the Spitzer data.

As expected from the description in $\S 3.1$, we found it difficult to determine a unique, "best" solution for the cyclotron model component. We determined the relative goodness of different model cyclotron components by calculating the $\chi^{2}$ and standard deviation of the residuals $\left(\sigma_{\text {res }}\right)$ in the $1-2.5 \mu \mathrm{m}$ wavelength region for the summed cyclotron, WD, and secondary star model components compared to the observed near-IR spectrum. Table 1 lists several "best" cyclotron models determined in this way for a range of $k T$ values. As $k T$ decreases, we had to decrease $B$ and increase $\Lambda$ in order for the cyclotron component to continue to match the wavelength spacing, widths, and relative peak amplitudes of the observed cyclotron humps. The goodness of the model also tends to decrease with decreasing $k T$, although none of the models has particularly poor agreement with the observed SED. In the end, for the purposes of this work, the specific cyclotron component that we use is not particularly important, since all of them contribute negligibly at mid-IR wavelengths. We will use the $k T=$ $10 \mathrm{keV}$ cyclotron model in the remainder of this work.
TABLE 1

Representative Cyclotron Models

\begin{tabular}{cccccccc}
\hline \hline $\begin{array}{c}k T \\
(\mathrm{keV})\end{array}$ & $\begin{array}{c}B \\
(\mathrm{MG})\end{array}$ & $\begin{array}{c}\theta \\
(\mathrm{deg})\end{array}$ & $\log \Lambda$ & $\log A$ & $\begin{array}{c}a_{\mathrm{cyc}} \\
\left(a_{\mathrm{wd}}\right)^{\mathrm{a}}\end{array}$ & $\chi^{2}$ & $\begin{array}{c}\sigma_{\text {res }} \\
(\mathrm{mJy})\end{array}$ \\
\hline $12 \ldots \ldots \ldots \ldots \ldots$. & 13.6 & 75 & 2.845 & -25.178 & 0.0145 & 0.202 & 0.0432 \\
$10 \ldots \ldots \ldots \ldots \ldots$. & 13.4 & 75 & 3.267 & -25.106 & 0.0172 & 0.224 & 0.0475 \\
$8 \ldots \ldots \ldots \ldots \ldots \ldots$. & 13.35 & 75 & 3.653 & -25.044 & 0.0198 & 0.424 & 0.0535 \\
$6 \ldots \ldots \ldots \ldots \ldots \ldots$ & 13.2 & 75 & 4.342 & -24.919 & 0.0264 & 0.728 & 0.0737 \\
\hline
\end{tabular}

${ }^{\mathrm{a}}$ The cyclotron emitting area $a_{\mathrm{cyc}}$, determined by the scale factor $A$, is given in units of the projected WD surface area $a_{\mathrm{wd}}=\pi R_{\mathrm{wd}}^{2}$.

In B07, we utilized a "sum-over-fields" approach to calculating the cyclotron component, which considered the summed contributions from cyclotron emission of electrons encountering a successively stronger magnetic field as they approached the WD. In part, this was an attempt to set a "worst case" limit for the contribution of cyclotron emission at long wavelengths (since the strength of cyclotron emission at long wavelengths is increased relative to short wavelengths through this approach). However, another effect of the sum-over-fields approach is to smear out the individual cyclotron humps, which is clearly inconsistent with the observed near-IR spectrum of EF Eri. Consequently, we have not used that approach here. In any case, even if we considered a two-part cyclotron component consisting of the single-field and summed-fields cases, the contribution of the latter would have to be extremely small in order not to dilute the strong observed single-field spectrum (and, regardless of strength, would not contribute significantly at $\lambda \gtrsim 4 \mu \mathrm{m}$ ).

\subsubsection{Comparison with Brinkworth et al. (2007)}

Figure $3 a$ shows the observational data from Figure 2 with a model containing an optically thick CBD similar to the best optically thick CBD component from B07 (see model 1 in Table 4 of that work). It has been adjusted slightly to account for the larger distance used here by changing the inner-edge temperature from 655 to $755 \mathrm{~K}$. The criterion used in B07 to best reproduce the $8 \mu \mathrm{m}$ IRAC point without exceeding any shorter wavelength point has been preserved. This model has the wrong spectral shape and significantly exceeds the longer wavelength SED of EF Eri that is revealed by our IRS spectrum.

\subsubsection{New Model Results}

Figure $3 b$ shows a new model that utilizes the additional constraints on flux density at long wavelengths provided by our IRS spectrum of EF Eri. The parameters common to this model and the one discussed below are listed in Table 2, while parameters specific to this model are listed in Table 3 (model 1). For this model, we have utilized an optically thin CBD composed of spherical dust grains that radiate as blackbodies according to the radial temperature profile as calculated for the optically thick CBD case (see $\S 3.1$ and B07).

This model shows significant improvement over that shown in Figure $3 a$, especially at the short- and long-wavelength ends. However, the model flux density is too low at the middle wavelengths (i.e., IRAC channels 1 and 2 at 3.6 and $4.5 \mu \mathrm{m}$ ). The total mass of the CBD is $\approx 10^{21} \mathrm{~g}$, consistent with the finding from $\mathrm{B} 07$ that the masses of CBDs in magnetic CVs are many orders of magnitude smaller than what is predicted to be required to influence the angular momentum loss history of these systems (Taam et al. 2003). For lower and higher inner-edge CBD temperatures, the overall flux density level of the observed SED can be matched 


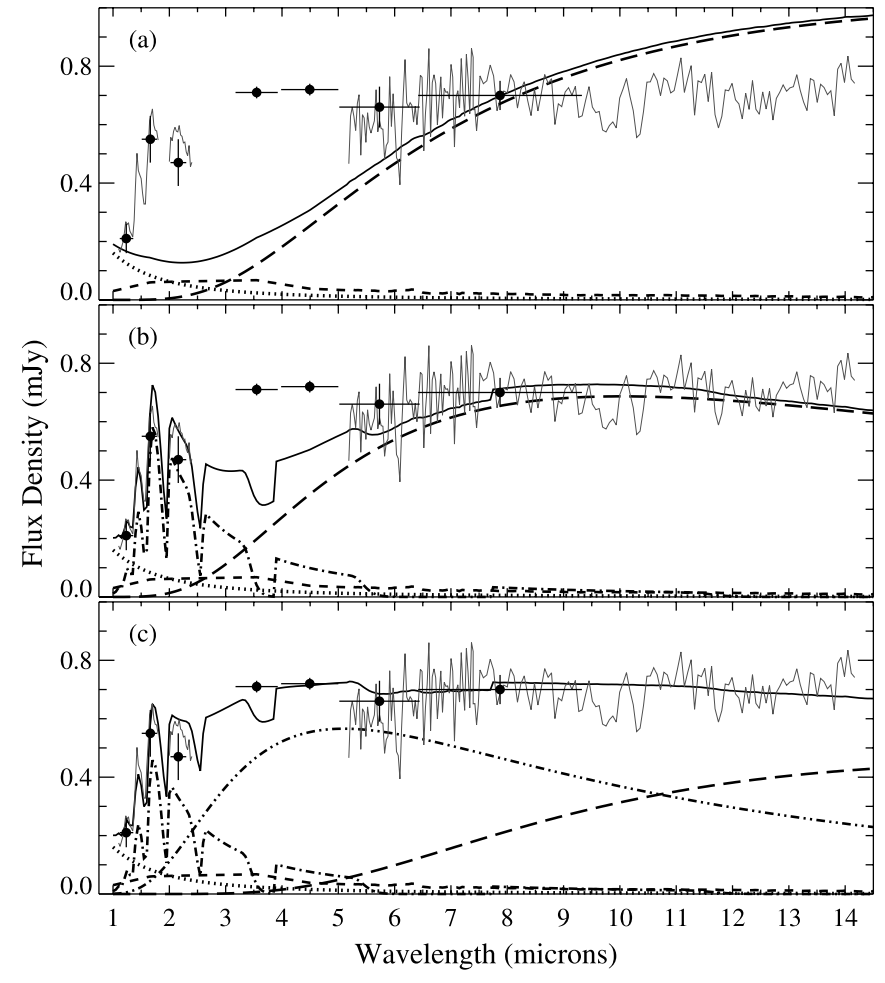

FIG. 3.-Mid-IR SED of EF Eri (from Fig. 2) with $a$ the optically thick CBD model, $b$ the new optically thin CBD and cyclotron model, and $c$ the new optically thin CBD and cyclotron model with an additional blackbody component. The model components are: WD (dotted line), secondary star (short-dashed line), circumbinary disk (long-dashed line), cyclotron emission (dot-dashed line), and blackbody (double-dot-dashed line); the total combined model is shown as a solid line. See text for additional details of the models. [See the electronic edition of the Journal for a color version of this figure.]

by increasing or decreasing, respectively, the total disk mass (i.e., the number of radiating dust grains). If the temperature of the inner edge of the CBD is decreased, then the match between the model and observed SEDs becomes worse; the CBD profile does not reach peak flux density until an even longer wavelength, which exacerbates the problem of missing flux density at the IRAC wavelengths. A higher temperature for the inner edge of the CBD produces a better match at the middle wavelengths. However, the model is then too faint at the long-wavelength end, since the CBD SED peaks and begins to decline at a shorter wavelength. (See $\S 3.2 .5$ for more discussion of the constraints on CBD model parameters.)

We have explored a possible means of reconciling this CBD model with the 3.6 and $4.5 \mu \mathrm{m}$ data. The model in Figure $3 c$ is similar to that shown in Figure $3 b$, but uses a blackbody component to account for the "missing" flux at 3.6 and $4.5 \mu \mathrm{m}$. The model parameters are listed in Tables 2 and 3 (model 2). This model has the advantage that it requires a CBD with a low inneredge temperature that could easily be produced by irradiation from the stellar components in EF Eri. On the other hand, it has the disadvantage that the physical origin of the additional component is unclear. The required equivalent emitting area (corresponding to a radius of $46 R_{\mathrm{wd}}$ ) is too large to be contained in the stellar Roche lobes, which points to the CBD. The required temperature is $1000 \mathrm{~K}$, which is somewhat uncomfortably warm from considerations of both the origin of the heating and the potential destruction of the dust grains. However, it might suggest that a more complex radial temperature profile in the CBD could produce an SED shape that is more consistent with the observations. We have not explored this possibility in detail, because it would introduce yet more free parameters into our already barely constrained model.

\subsubsection{Uniqueness, Plausibility, and Constraints of the Circumbinary Disk Models}

Much like the cyclotron emission component (see $\S \S 3.1$ and 3.2.2), the model CBD SEDs shown in this work are not, strictly speaking, unique solutions, in the sense that very similar results can be achieved from somewhat different combinations of input parameters. We have tried to minimize this as much as possible by constraining plausible parameter ranges based on whatever other information, observational data, and reasonable assumptions are available. In this section, we describe in detail the justification for the constraints that have been assumed in fixing the exponent in the radial temperature profile calculation (see $\S 3.1$ ) and the inner radius, $R_{\text {in }}$, of the model CBD. We also expand on

TABLE 2

Shared Model Parameters

\begin{tabular}{|c|c|c|c|}
\hline Component & Parameter & Value & References \\
\hline \multirow[t]{3}{*}{  } & Orbital period, $P_{\text {orb }}$ (minutes) & $81.022932(8)$ & 1 \\
\hline & Inclination, $i$ (deg) & $55(5)$ & 1 \\
\hline & Distance, $d(\mathrm{pc})$ & 132 & 2 \\
\hline \multirow[t]{3}{*}{ WD } & Temperature, $T_{\mathrm{wd}}(1000 \mathrm{~K})$ & $9.5(0.5)$ & 3 \\
\hline & Mass, $M_{\mathrm{wd}}\left(M_{\odot}\right)$ & 0.6 & 3 \\
\hline & Radius, $R_{\mathrm{wd}}\left(R_{\odot}\right)$ & 0.0125 & 4 \\
\hline \multirow[t]{5}{*}{ SS } & Template star & 2MASS J15074769-1627386 & \\
\hline & Spectral type & L5 & 4,5 \\
\hline & Temperature, $T_{2}(1000 \mathrm{~K})$ & 1.7 & 4 \\
\hline & Mass, $M_{2}\left(M_{\odot}\right)$ & 0.055 & 6 \\
\hline & Radius, $R_{2}\left(R_{\odot}\right)$ & 0.1 & 4 \\
\hline \multirow[t]{6}{*}{ CBD } & Optical depth prescription & Thin & \\
\hline & Temperature profile exponent & 0.75 & \\
\hline & Disk annulus treatment & Equal mass & \\
\hline & Constant height, $h_{\mathrm{cbd}}\left(R_{\mathrm{wd}}\right)$ & 0.00167 & \\
\hline & Grain density, $\rho_{\text {grain }}\left(\mathrm{g} \mathrm{cm}^{-3}\right)$ & 3.0 & \\
\hline & Grain radius, $r_{\text {grain }}(\mu \mathrm{m})$ & 1 & \\
\hline
\end{tabular}

References.-(1) Piirola et al. 1987; (2) Thorstensen 2003; (3) Beuermann et al. 2000; (4) Brinkworth et al. 2007, and references therein; (5) Howell \& Ciardi 2001; (6) Howell et al. 2006a. 
TABLE 3

Model-Specific Parameters

\begin{tabular}{|c|c|c|c|}
\hline \multirow[b]{2}{*}{ COMPONENT } & \multirow[b]{2}{*}{ PARAMETER } & \multicolumn{2}{|c|}{ VALUE } \\
\hline & & Model 1 & Model 2 \\
\hline \multirow[t]{8}{*}{ CYC .................... } & WD magnetic field, $B$ (MG) & 13.4 & 13.4 \\
\hline & Electron temperature, $k T(\mathrm{keV})$ & 10 & 10 \\
\hline & Angle to magnetic field, $\theta$ (deg) & 75 & 75 \\
\hline & Size parameter, $\log \Lambda$ & 3.267 & 3.301 \\
\hline & Scale factor, $\log A$ & -25.106 & -25.220 \\
\hline & Emitting area, $a_{\mathrm{cyc}}\left(a_{\mathrm{wd}}\right)$ & 0.0172 & 0.0132 \\
\hline & Model goodness, $\chi^{2}$ & 0.224 & 0.286 \\
\hline & Model residuals, $\sigma_{\text {res }}(\mathrm{mJy})$ & 0.0475 & 0.0522 \\
\hline \multirow[t]{5}{*}{ CBD $\ldots \ldots \ldots \ldots \ldots$} & Inner-edge temperature, $T_{\text {in }}(\mathrm{K})$ & 830 & 450 \\
\hline & Inner-edge radius, $R_{\text {in }}\left(R_{\mathrm{wd}}\right)$ & 73 & 73 \\
\hline & Outer-edge temperature, $T_{\text {out }}(\mathrm{K})$ & 20 & 20 \\
\hline & Outer-edge radius, $R_{\text {out }}\left(R_{\mathrm{wd}}\right)$ & 10482 & 4634 \\
\hline & Total mass, $M_{\mathrm{cbd}}\left(10^{20} \mathrm{~g}\right)$ & 9.55 & 17.3 \\
\hline \multirow[t]{2}{*}{ 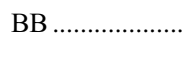 } & Temperature, $T(\mathrm{~K})$ & $\ldots$ & 1000 \\
\hline & Radius of emitting area, $R_{\mathrm{bb}}\left(R_{\mathrm{wd}}\right)$ & $\ldots$ & 45.6 \\
\hline
\end{tabular}

the discussion of the failure of the optically thick CBD model first mentioned in $\S 3.2 .3$.

In general terms, the influence of the exponent in the radial temperature profile can be described as follows: a larger (smaller) exponent in the CBD radial temperature profile leads to a steeper (shallower) temperature gradient near the inner edge of the disk, and overall lower (higher) temperature throughout the disk, which corresponds to large (small) dust grains that do (do not) cool efficiently. In practice, we have found that an exponent of 3/4, as used here and in B07, produces the most viable results in comparison with our observations of CVs. Smaller exponents produce CBD SEDs that rise too steeply and are, overall, too bright to reproduce the observed SEDs without arbitrarily increasing the distances to the CVs to many hundreds or thousands of pc. Larger exponents produce CBD SEDs that rise too shallowly at longer wavelengths and are, overall, too faint to match the observed mid-IR flux densities without arbitrarily increasing the temperature of the disk's inner edge to unrealistic levels. For example, we can obtain a model optically thin CBD SED that is essentially indistinguishable from that in model 1 (Fig. $3 b$ ) by using a larger radial temperature profile exponent ( 1 instead of $3 / 4$; see $\S 3.1$ ) and a smaller total disk mass $\left(4.41 \times 10^{20} \mathrm{~g}\right.$ instead of $\left.9.55 \times 10^{20} \mathrm{~g}\right)$; however, one objection to this approach is that departure from an exponent of 3/4 implies dust grains that are not blackbodies (Frank et al. 2002), whereas this is an implicit assumption of the CBD model calculations.

The inner-edge radius of the CBD models calculated in this work (for both optically thick and thin cases) is fixed at $R_{\text {in }}=$ $73 R_{\mathrm{wd}}$, which is a lower limit set by the tidal truncation radius of EF Eri. Increasing the inner radius of the CBD from this value worsens the agreement between the CBD models and the observed SED, because the irradiation-induced temperature of the inner edge will then be lower. This effectively removes flux from the short IR wavelengths of the model SED, whereas the main problem we have in reproducing the observations is that the models already have too little flux at short IR wavelengths. (The outer radius of the CBD is calculated to correspond to a temperature of $20 \mathrm{~K}$ according to the radial temperature profile in use, but at wavelengths shortward of $15 \mu \mathrm{m}$, the resultant profile is insensitive to increasing the temperature of the outer-radius cutoff by as much as an order of magnitude.) Even if we arbitrarily (and unphysically) decrease the inner-edge radius of the CBD to $50 R_{\mathrm{wd}}$, which is barely larger than the distance from the CV's center of mass to the back of the secondary star's Roche lobe $\left(49 R_{\mathrm{wd}}\right)$, the resultant model CBD SED (for both optically thick and thin cases) has only a few percent improvement (increase) in flux density at IRAC channels 1 and 2. Similarly, the agreement with the IRAC channels 3 and 4 and IRS data is not significantly better than that achieved by the CBD component used in model 1 (Fig. 3b).

The optically thick CBD models are parameterized solely by the radial temperature profile (including the value of $T_{\text {in }}$ ) and size (i.e., inner and outer radii). As such, the variety of possible model optically thick CBD SEDs is rather more limited than for the optically thin case. Changing either the radial temperature profile exponent or $R_{\text {in }}$ does not yield an optically thick CBD model that reproduces the observed SED any better than the model shown in Figure $3 a$ and discussed in $\S 3.2 .3$. For example, using an exponent of 1 , we can produce an optically thick CBD SED whose shape (i.e., relative intensity at each wavelength) is almost indistinguishable from that of the optically thin model CBD SED in model 1 . However, it is overall $15 \%-30 \%$ fainter than the optically thin model, and so produces much worse agreement with the observations. Smaller exponents produce model CBD SEDs that are far too bright, especially at longer wavelengths. Increasing $T_{\text {in }}$ for the optically thick CBD produces more flux at shorter wavelengths, but also increases the steepness of the SED and the excess flux at longer wavelengths. Decreasing $T_{\text {in }}$ begins to flatten the SED, but also makes it overall too faint at all wavelengths, especially the short IR wavelengths. Increasing $R_{\text {in }}$ also fails, since (as described above) this results in even less flux at the short IR wavelengths. In light of the flat shape of the EF Eri SED at wavelengths longer than the IRAC regime, we conclude from the behavior of the optically thick CBD models described in this section that they are much less likely than the more flexible optically thin CBD models as viable explanations of the mid-IR SED of EF Eri.

\section{CONCLUSIONS}

Our newly obtained mid-IR spectrum of EF Eri has allowed us to further constrain and refine the SED model for this system first presented in B07. Based on the B07 model, which was constrained by only $2 \mathrm{MASS}$ and IRAC photometric data, we would have expected the 8-14 $\mu \mathrm{m}$ SED of EF Eri to either rise (if dominated by an optically thick CBD) or fall (if dominated by shortwavelength cyclotron emission). However, the observed spectrum defied both of our expectations by remaining almost flat compared to the IRAC data. This does allow us to eliminate cyclotron emission as a dominant component in the mid-IR SED of EF Eri beyond $\lambda \approx 3 \mu \mathrm{m}$. At the same time, we also show that a geometrically thin, but optically thick, CBD is unlikely as a viable explanation for the spatial distribution of dust in EF Eri. Instead, the dust is more likely present as a geometrically and optically thin CBD. In all cases, however, there are inconsistencies between our CBD models and the observed SED of EF Eri at the IRAC channel 1 and 2 wavelengths $(3.6$ and $4.5 \mu \mathrm{m})$, which imply that a more complex situation is present than represented by our simple CBD models. As also found in B07, the total mass of dust in the CBD is still several orders of magnitude too small to strongly affect the secular evolution of CVs in the context of current models of angular momentum loss mechanisms in CVs.

Based on our study of EF Eri, we can make several generalizations regarding the mid-IR observational properties of CVs that contain dust. First, EF Eri is a low-field polar. In polars with moderate to strong WD magnetic fields of several tens of MG or more, cyclotron emission will be shifted to even shorter wavelengths and be even less important for understanding the mid-IR SED. Second, EF Eri contains a very low-mass, faint secondary star. 
Longer orbital period systems will have correspondingly more massive, brighter secondary stars. However, in the IRAC bands, an M5 dwarf is only $\approx 10$ times brighter than an L5 brown dwarf (Patten et al. 2006). Although the observed $3.6 \mu \mathrm{m}$ flux density in EF Eri is comparable to that of an M5 star, at $8 \mu \mathrm{m}$ the observed flux density is $\approx 4$ times that of an M5 star. So, even in systems containing a more massive secondary star, dust emission at a comparable level to that in EF Eri produces a mid-IR SED far in excess of the stellar components. Finally, EF Eri lacks an accretion disk. In nonmagnetic CVs, the hot accretion disk will appear in the IR as a Rayleigh-Jeans-like tail similar in shape to the WD SED, but will likely be significantly brighter than both stellar components. However, the maximum possible emitting area for the accretion disk is limited by the size of the WD Roche lobe. The CBD, on the other hand, can have an emitting area many orders of magnitude larger. Hence, even in the presence of an accretion disk, the system SED at the longest mid-IR wavelengths could still be dominated by dust emission.

An extrapolation of our current CBD model for EF Eri to even longer wavelengths predicts a continued gradual decline in the overall flux density, with (for example) the total flux density of EF Eri at $24 \mu \mathrm{m}$ being about $90 \%$ of the $8 \mu \mathrm{m}$ value. Longer wavelength observations (e.g., at the Spitzer Peak-up Imaging $22 \mu \mathrm{m}$ or MIPS $24 \mu \mathrm{m}$ bands) could test this prediction.

This work is based in part on observations made with the Spitzer Space Telescope, which is operated by the Jet Propulsion Laboratory, California Institute of Technology, under a contract with NASA. Support for this work was provided by NASA. We thank the Spitzer Science Center (SSC) Director for his generous allocation of observing time for the NASA/NOAO/Spitzer Space Telescope Observing Program for Students and Teachers. The National Optical Astronomy Observatory (NOAO), which is operated by the Association of Universities for Research in Astronomy (AURA), Inc., under cooperative agreement with the National Science Foundation (NSF), has provided many in kind contributions for which S. B. H. is grateful. This work makes use of data products from the Two Micron All Sky Survey, which is a joint project of the University of Massachusetts and the Infrared Processing and Analysis Center/Caltech, funded by NASA and the NSF. C. S. B. acknowledges support from the SSC Enhanced Science Fund and NASA's Michelson Science Center. D. W. H. thanks Axel Schwope for helpful advice on calculating cyclotron spectra.

Beuermann, K., Euchner, F., Reinsch, K., Jordan, S., Gänsicke, B. T. 2007, A\&A, 463, 647

Beuermann, K., Wheatley, P., Ramsay, G., Euchner, F., \& Gänsicke, B. T. 2000, A\&A, 354, L49

Brinkworth, C. S., et al. 2007, ApJ, 659, 1541 (B07)

Chanmugam, G. 1980, ApJ, 241, 1122

Cushing, M. C., et al. 2006, ApJ, 648, 614

Fazio, G., et al. 2004, ApJS, 154, 10

Frank, J., King, A., \& Raine, D. 2002, in Accretion Power in Astrophysics (Cambridge: Cambridge Univ. Press), Chap. 5

Harrison, T. E., Campbell, R. K., Howell, S. B., Cordova, F. A., \& Schwope, A. D. 2007, ApJ, 656, 444

Harrison, T. E., Howell, S. B., Szkody, P., Homeier, D., Johnson, J., \& Osborne, H. 2004, ApJ, 614, 947

Houck, J. R., et al. 2004, ApJS, 154, 18

Howell, S. B., \& Ciardi, D. R. 2001, ApJ, 550, L57

Howell, S. B., Nelson, L., \& Rappaport, S. 2001, ApJ, 550, 897

Howell, S. B., Walter, F. M., Harrison, T. E., Huber, M. E., Becker, R. H., \& White, R. L. 2006a, ApJ, 652, 709

Howell, S. B., et al. 2006b, ApJ, 646, L65

Patten, B. M., et al. 2006, ApJ, 651, 502

Piirola, V., Coye, G. V., \& Reiz, A. 1987, A\&A, 186, 120

Reid, I. N., Kirkpatrick, J. D., Gizis, J. E., Dahn, C. C., Monet, D. G., Williams, R. J., Liebert, J., \& Burgasser, A. J. 2000, AJ, 119, 369

Schwope, A. D., Beuermann, K., \& Thomas, H.-C. 1990, A\&A, 230, 120

Schwope, A. D., Staude, A., Koester, D., \& Vogel, J. 2007, A\&A, 469, 1027

Szkody, P., Harrison, T. E., Plotkin, R. M., Howell, S. B., Seibert, M., \& Bianchi, L. 2006, ApJ, 646, L147

Taam, R. E., Sandquist, E. L., \& Dubus, G. 2003, ApJ, 592, 1124

Thompson, A. M., \& Cawthorne, T. V. 1987, MNRAS, 224, 425

Thorstensen, J. R. 2003, AJ, 126, 3017

Warner, B. 1995, Cataclysmic Variable Stars (Cambridge: Cambridge Univ. Press)

Werner, M., et al. 2004, ApJS, 154, 1

Wheatley, P. J., \& Ramsay, G. 1998, in ASP Conf. Ser. 137, Wild Stars in the Old

West, ed. S. Howell, E. Kuulkers, \& C. Woodward (San Francisco: ASP), 446 